\title{
Experimental Study of the Rheological Behaviors of Fresh Concrete, Mortar, and Clay Grout
}

\author{
Cho-Liang Tsai \\ Department of Construction Engineering National Yunlin University of Science and Technology, 123, Sec. III, University \\ Rd., Touliu, Taiwan 640,, tsaicl@ce.yuntech.edu.tw \\ Yun Du \\ Graduate School of Engineering Sci. \& Tech., National Yunlin University of Sci. \& Tech; Department of Civil \\ Engineering, Dahan Institute of Technology, Hualian, Taiwan 971.
}

Yi-Shiun Tsai

General Education Center, Hsiuping Institute of Technology, Taichung, Taiwan 412.

Follow this and additional works at: https://jmstt.ntou.edu.tw/journal

Part of the Civil and Environmental Engineering Commons

\section{Recommended Citation}

Tsai, Cho-Liang; Du, Yun; and Tsai, Yi-Shiun (2003) "Experimental Study of the Rheological Behaviors of Fresh Concrete, Mortar, and Clay Grout," Journal of Marine Science and Technology. Vol. 11: Iss. 3, Article 1.

DOI: $10.51400 / 2709-6998.2270$

Available at: https://jmstt.ntou.edu.tw/journal/vol11/iss3/1

This Research Article is brought to you for free and open access by Journal of Marine Science and Technology. It has been accepted for inclusion in Journal of Marine Science and Technology by an authorized editor of Journal of Marine Science and Technology. 


\title{
EXPERIMENTAL STUDY OF THE RHEOLOGICAL BEHAVIORS OF FRESH CONCRETE, MORTAR, AND CLAY GROUT
}

\author{
Cho-Liang Tsai*, Yun Du**, and Yi-Shiun Tsai***
}

Key words: rheological property, fresh concrete, mortar, clay grout.

\begin{abstract}
Most studies assume that fresh concrete is a Bingham fluid, but few studies precisely measure its Bingham constants, shear yield stress and viscosity, especially for normal fresh concrete. In stead of using commercial viscometer, this work developed a new experimental method and analytical procedure, based on the Stokes' flow, for characterizing rheological properties of fluids. The experimental method developed here involved dragging a sphere submerged in the specimen fluid at a constant speed and measuring the resistance. Rheological properties of the specimen fluid can be calculated from the theoretical function correlating the drag velocity, drag force, and rheological properties of the fluid.

Clay grout, fresh mortar and normal fresh concrete were tested. The measured Bingham constants were repeatable and stable, and importantly revealed that the clay grout and fresh mortar are Bingham fluids with very low viscosity, while normal fresh concrete is a Bingham fluid with rheological properties that depend on flow rate. This study also verifies that the shear stress required to start a concrete flow exceeds that required for maintaining it.
\end{abstract}

\section{INTRODUCTION}

For over 25 years fresh mortar and concrete have been considered approximately Bingham materials that can be characterized by shear yield stress and viscosity. In Bingham model, the relationship between applied shear stress $(\tau)$ and rate of shear strain $(j)$ is near-linear and has a positive intercept on the stress axis [2] (Fig. 1).

$$
\tau=\tau_{o}+\mu \dot{\gamma}
$$

Paper Submitted 04/14/03, Accepted 08/1 1/03. Author for Correspondence: Cho-Liang Tsai.

*Department of Construction Engineering National Yunlin University of Science and Technology, 123, Sec. III, University Rd., Touliu, Taiwan 640, E-mail: tsaicl@ce.yuntech.edu.tw.

**Graduate School of Engineering Sci. \& Tech., National Yunlin University of Sci. \& Tech; Department of Civil Engineering, Dahan Institute of Technology, Hualian, Taiwan 971.

***General Education Center, Hsiuping Institute of Technology, Taichung, Taiwan 412 . where $\tau_{o}$ and $\mu$ denote the Bingham constants: shear yield stress and plastic viscosity respectively. When the applied pressure or force induces sufficient shear stress $\tau$ to overcome the shear yield stress $\tau_{o}$, Bingham flow occurs and the rate of the shear strain in the flow is proportional to $\tau-\tau_{o}$. Although some researchers are still uncomfortable about this assumption [3], various fixtures have been developed based on this mathematic model for measuring the constants $[2,3,4,6,8,12,13]$.

\section{Flow-cone Method [8]}

Cone-type viscometers, such as the Engler viscometer, are used to measure the rheological properties of Newtonian fluids and Bingham fluids [4]. In some countries, some types of flow-cone are the standard for testing the cement grout, used for prepacked and prestressed concrete, which is generally recognized as approximating a Bingham material. Among the flowcones, the P-type flow-cone is simple and commonly used [4]. The P-type flow-cone consists of two parts, a funnel and a discharge tube (Fig. 2).

Theoretical analysis reveals that when the funnel is filled with fluid its Bingham constants are related to the flow velocity in the discharge tube, and can be calculated if the flow velocity is measured.

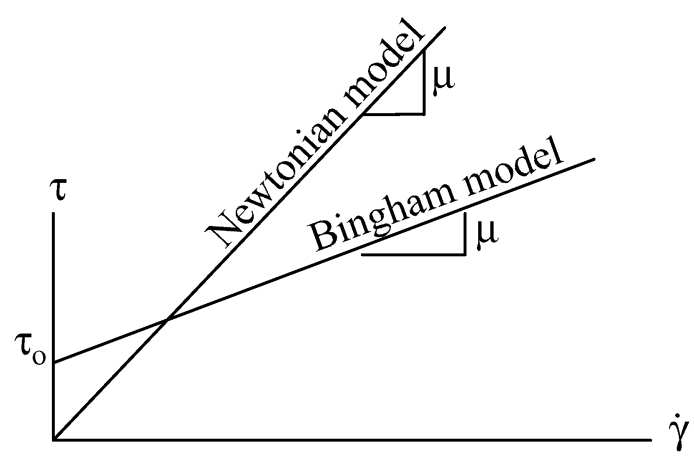

Fig. 1. The relationship between the applied shear stress $(\tau)$ and the resulting rate of strain $(j)$ in Bingham and Newtonian models of fluid. 


\section{Inclined-pipe Testing Method [8]}

The inclined-pipe is a modification of the flowcone. This pipe has a bent funnel top, and an overflow opening to ensure a constant fluid depth in the funnel. Moreover, the diameter of the top of the funnel is ten times that of the discharge tube, ensuring sufficiently low fluid flow velocity to be neglected at the top of the funnel compared with inside the discharge tube. Discharge tube inclination $\theta$ is variable (Fig. 3). The flow rate through the inclined pipe can be measured experimentally to calculate the viscosity and the shear yield stress of the fluid.

\section{Pipe Flow Method [12]}

This method was developed based on Poiseuille flow equation for Bingham materials which indicates that the difference between the pressures of the flowing fluid at two cross sections in a pipe with constant cross section is a function of the $t_{o}$ and $\mu$ of the fluid (Fig. 4). A better method of obtaining $P$ is applying strain gage to the outer surface of the pipe to measure hoop strain, which is proportional to the fluid pressure inside the pipe.

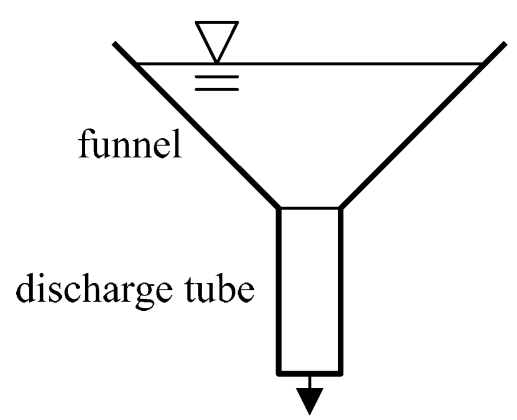

Fig. 2. A flow cone.

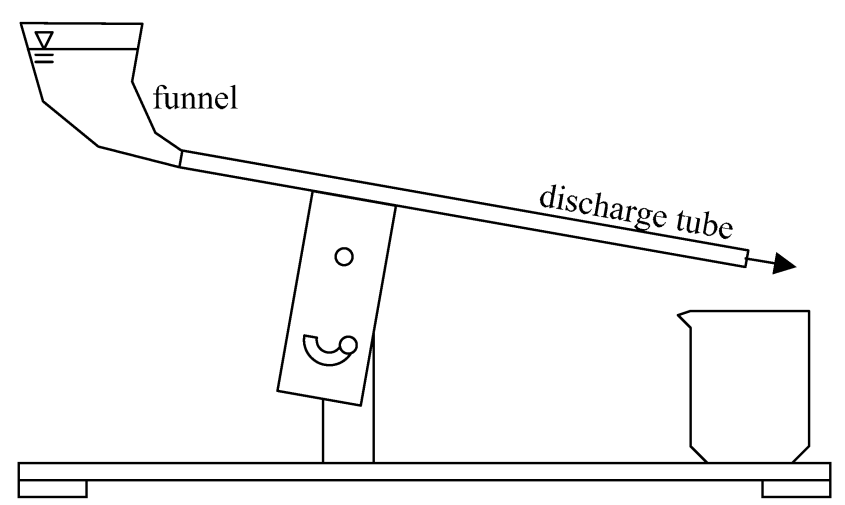

Fig. 3. Inclined-pipe testing apparatus.

\section{Rotary Duplex Cylinder Viscometer [13]}

A rotary duplex cylinder viscometer, comprising a sample apparatus, a stress controller, and a data acquisition system, is used to characterize shear yield stress and plastic viscosity (Fig. 5). The sample apparatus includes an internal rotator and an external sleeve. The dimensions of the internal rotator and the external sleeve can be selected according to rheological properties of the fluid specimen. The stress controller controls the torque of the internal rotator. If the relationship between the torque and the rotational speed is linear, the coefficients of the linearity are preoperational to the Bingham constants of the fluid.

\section{Rotary Shaft Viscometer $[2,3]$}

Another type of viscometer, similar to the rotary duplex cylinder viscometer, was developed. The internal rotator of the rotary duplex cylinder viscometer is replaced by a shaft, with or without blades (Fig. 6). Several types of shaft are developed for fluids with various properties. The same principle as that of rotary duplex cylinder viscometer is employed to interpret the data. Fluids with known properties are required to

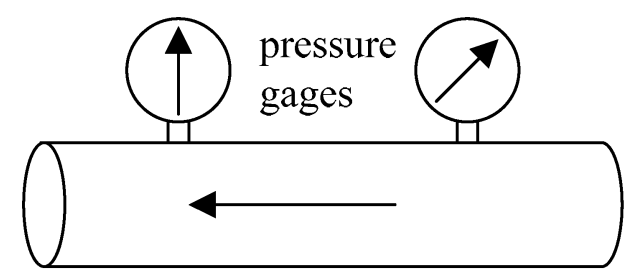

Fig. 4. Tube flow testing apparatus.

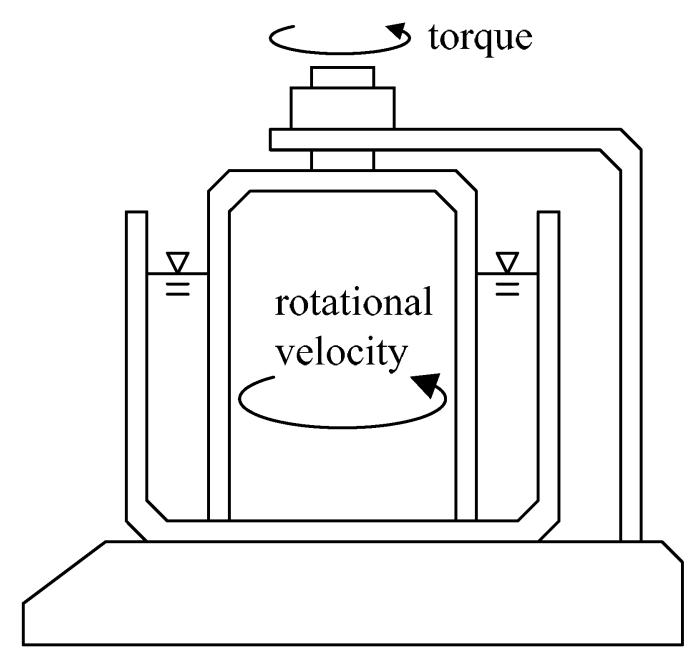

Fig. 5. Rotary duplex cylinder viscometer. 
characterize the system to make it works. The rotary shaft viscometer is also very easy to be operated, and works for most of the homogeneous fluids. However, the experimental results for fresh concrete are as unstable as, if not worse than, those from rotary duplex cylinder viscometer, because the coarse aggregate hits and scratches the shaft, blades and container during the experiment.

Notably, some commercial viscometers employ empirical or semi-empirical function. Nominal values corresponding to the rheological properties of the fluid specimen can be measured. The system first must be characterized using characterizing fluids to determine the relationship between the measured nominal values and desired rheological properties of the specimen. Obviously, the characterizing fluids must be characterized using other methods that can identify its rheological properties directly, such as the flow-cone, inclinedpipe testing, and tube flow methods. Unfortunately, these methods are usually ineffective for aggregated fluids. The characterizing fluids are usually Newtonian fluids, such as clear homogeneous oil, and have quite different properties to of fresh concrete. The question thus arises of how a system that is characterized only by Newtonian fluids, as are most of the viscometers, can be effectively measure the rheological properties of Bingham fluids. The rheological properties of aggregated fluids such as fresh mortar and concrete are closely related to the state of flocculation state of their aggregate. Attractive interparticle forces or settlement drive the aggregate to form a temporary internal structure, which is why some initial shear stress is required to overcome the yielding shear stress, destroy the temporary internal structure, and make the fluid thixotropic. Additionally, it seems unscientific to measure the rheological properties of such highly heterogeneous aggregated fluids in a

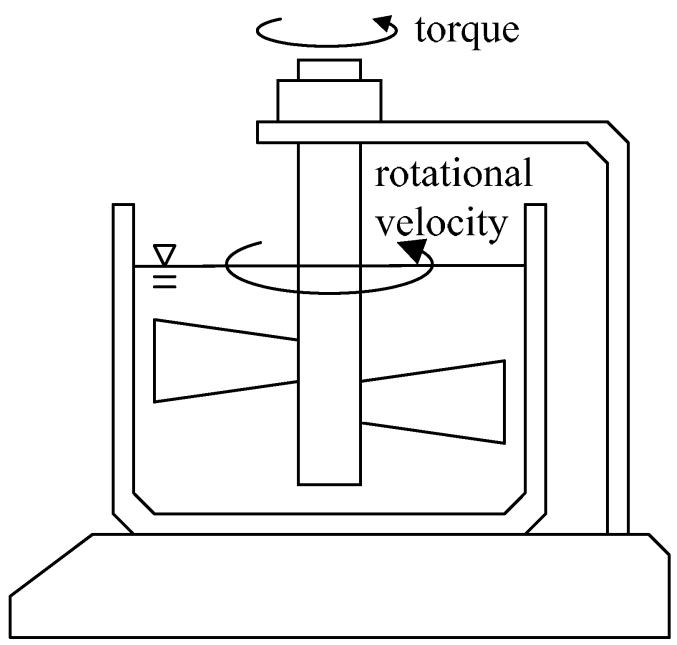

Fig. 6. Rotary shaft viscometer. narrow container, tube or pipe, as most conventional viscometers do.

Some of the various methods for studying rheological properties of fluids work for mortar, while others work high-performance fresh concrete. Most such methods are barely satisfactory for normal fresh concrete. This study develops a new method that effectively characterizes normal fresh concrete and mortar directly based on Stokes' flow [1].

\section{STOKES' FLOW THEORY [9]}

In 1851, Stokes solved the problem of Newtonian flow pushing a fixed sphere (Fig. 7).

$$
D=6 \pi a \mu v
$$

where $D$ denotes the pushing force on the flow to the sphere, a represents sphere radius, $\mu$ is viscosity of the fluid, and $v$ is flow velocity. The solution also holds for the counterpart of this problem, a solid sphere being dragged in fluid at a constant velocity $v$. In this case, $D$ represents the drag force. For Bingham flow, the drag force induced by the yield shear stress of the fluid $\tau_{o}$, should be added to Eq. (2), producing,

$$
D=6 \pi a \mu v+\oint_{s} \tau_{o} \sin \alpha d s=6 \pi a \mu v+\pi^{2} a^{2} \tau_{o}
$$

where the $\mu$ and $\tau_{0}$ are constants, $s$ denotes the sphere surface, and $\alpha$ represents the angle between the movement direction of the sphere (Fig. 7) and the normal direction of the sphere's surface. The drag coefficient, $C_{D}$, is defined as,

$$
D=C_{D} \rho \frac{v^{2}}{2} A
$$

where $\rho$, denotes the unit mass of the fluid, and $A$ represents the frontal area of the sphere normal to $v, A$ $=\pi a^{2} \cdot C_{D}$ can be derived as,

$$
C_{D}=\frac{D}{\rho \frac{v^{2}}{2} \pi a^{2}}=\frac{6 \pi a \mu v+\pi^{2} a^{2} \tau_{o}}{\rho \frac{v^{2}}{2} \pi a^{2}}
$$

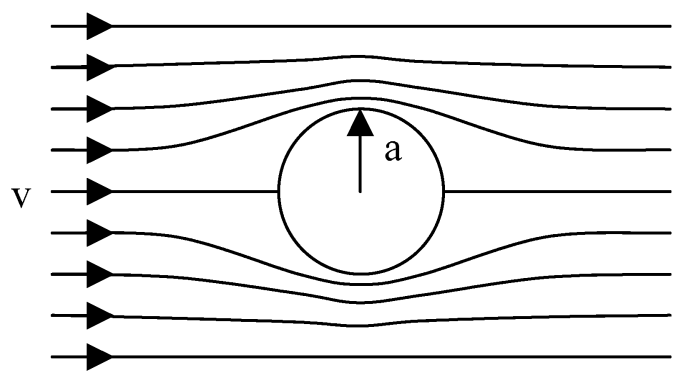

Fig. 7. Streamlines for Stokes' flow past a fixed sphere. 
Let $C_{D}=24 / R$, and let $R$ be the Reynolds number [9]. Then,

$$
R=\frac{12 \rho v^{2} \pi a^{2}}{D}=\frac{12 \rho v^{2} \pi a^{2}}{6 \pi a \mu \nu+\pi^{2} a^{2} \tau_{o}}
$$

If this Reynolds number exceeds one, then the fluid inertia can no longer be neglected and Stokes' solution does not hold. Under this condition, the velocity, $v$, must be limited. Maltose was tested to establish confidence in this equation. When a steel spherical pill was placed into maltose, it began to sink, and the downward drag force $D$ was the weight of the pill minus the buoyant force.

$$
\begin{aligned}
& D=W-\frac{4}{3} \pi a^{3} \rho G=\frac{4}{3} \pi a^{3}\left(\rho_{s}-\rho\right) G \\
& a=\left[\frac{3 D}{4 \pi\left(\rho_{s}-\rho\right) G}\right]^{1 / 3}
\end{aligned}
$$

where $W$ and $\rho_{s}$ denote the weight and unit mass, respectively, of the spherical pill. Combining of Eqs. $(3,7,8)$ to show the relationship between $D, v, \mu$ and $\tau_{o}$.

$$
\begin{aligned}
D & =6 \pi\left[\frac{3}{4 \pi\left(\rho_{s}-\rho\right) G}\right]^{1 / 3} D^{1 / 3} \mu \nu \\
& +\pi^{2}\left[\frac{3}{4 \pi\left(\rho_{s}-\rho\right) G}\right]^{2 / 3} D^{2 / 3} \tau_{o}
\end{aligned}
$$

where $v$ is the terminal velocity. The Reynolds number can also be expressed as,

$$
R=\frac{12 \rho v^{2} \pi a^{2}}{D}=\frac{12 \rho v^{2} \pi a^{2}}{W-\frac{4}{3} \pi a^{3} \rho G}
$$

In Eq. (9), $v$ can be measured from periodically photographs of the pill showing its locations in the transparent maltose, and $D$ can be calculated from Eq. (7). The terminal velocities corresponding to pills with various radii and weights were measured (Fig. 8). Next, $\mu$ and $\tau_{o}$ were calculated from Eq. (9) by least squares

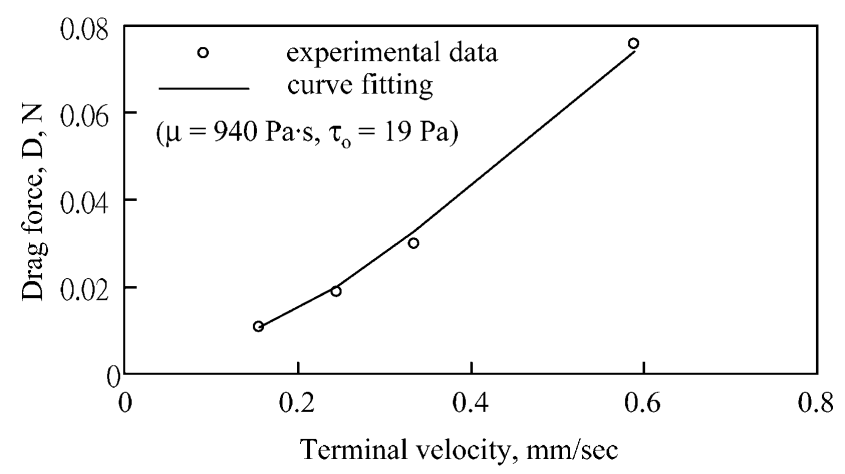

Fig. 8. The drag force vs. the terminal velocity of a sphere falling in maltose. curve fitting based on the data of $(D, v) . \mu$ and $\tau_{o}$ were $940 \mathrm{~Pa} \cdot \mathrm{Psec}$ and $19 \mathrm{~Pa}$, respectively. Excellent fitting was noted. The Reynolds number in Eq. (10) was in the order of $10^{-5}$ to $10^{-6}$ in this experiment. Maltose is sensitive to temperature, which was $25^{\circ} \mathrm{C}$ in this experiment. The $\tau_{o}$ of maltose appears very small, indicating that maltose is close to a Newtonian fluid. This method appears very effectively for characterizing the rheological properties of maltose.

\section{EXPERIMENTAL PROCEDURE}

In some cases, fluids such as clay grout, fresh mortar and fresh concrete are not transparent. Such cases necessitate modifying the experimental process. Instead of measuring the terminal velocity of a spherical pill sinking in the fluid, a sphere submerged in the fluid was dragged upwards by a thin drag wire at a controlled speed. The effect of the thin drag wire was neglected. The container holding the fluid specimen was a $50 \mathrm{~cm} \phi \times 100 \mathrm{~cm}$ bucket. The radius of the sphere was $25.4 \mathrm{~mm}$ or $34.1 \mathrm{~mm}$. A universal testing machine was used to pull the wire attached to the sphere and measure the drag force (Fig. 9). Clay grout, fresh mortar and normal fresh concrete were tested. The water concentration of the clay grout was $104 \%$ by weight. Weight ratios of water, cement, and fine aggregate in the mortar were $14.4 \%, 23.2 \%, 62.2 \%$. Fineness modulus of the fine aggregate used in the mortar and cement was 3.1. Weights of cement, water, coarse aggregate, and fine aggregate per unit concrete were $366 \mathrm{~kg} / \mathrm{m}^{3}, 226.92 \mathrm{~kg} / \mathrm{m}^{3}, 685.02 \mathrm{~kg} / \mathrm{m}^{3}$, and $976.59 \mathrm{~kg} / \mathrm{m}^{3}$. The maximum size of the coarse aggregate used in the concrete was $9.5 \mathrm{~mm}(3 / 8 ")$. Table 1 lists the weight ratio percentages of each grain size in

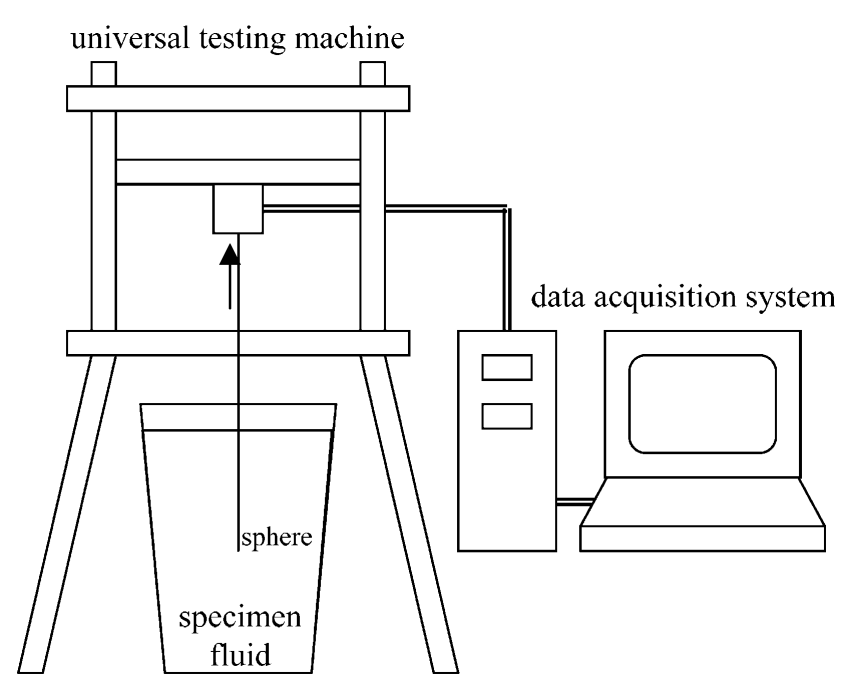

Fig. 9. Testing system for rheological properties of fluids. 
Table 1. Weight ratio percentages of each grain size in coarse aggregate and sand

\begin{tabular}{lccrrrrrrrrrc}
\hline U.S. standard sieve size & $37.5 \mathrm{~mm}$ & $25 \mathrm{~mm}$ & $19 \mathrm{~mm}$ & $12.5 \mathrm{~mm}$ & $\# 4$ & $\# 8$ & $\# 16$ & $\# 30$ & $\# 50$ & $\# 100$ & $\# 200$ & pan \\
\hline coarse aggregate & 0 & 0 & 20 & 60 & 9 & 9 & 1 & 1 & 0 & 0 & 0 & 0 \\
sand & 0 & 0 & 0 & 0 & 0 & 16 & 28 & 21 & 16 & 12 & 5 & 2 \\
\hline
\end{tabular}

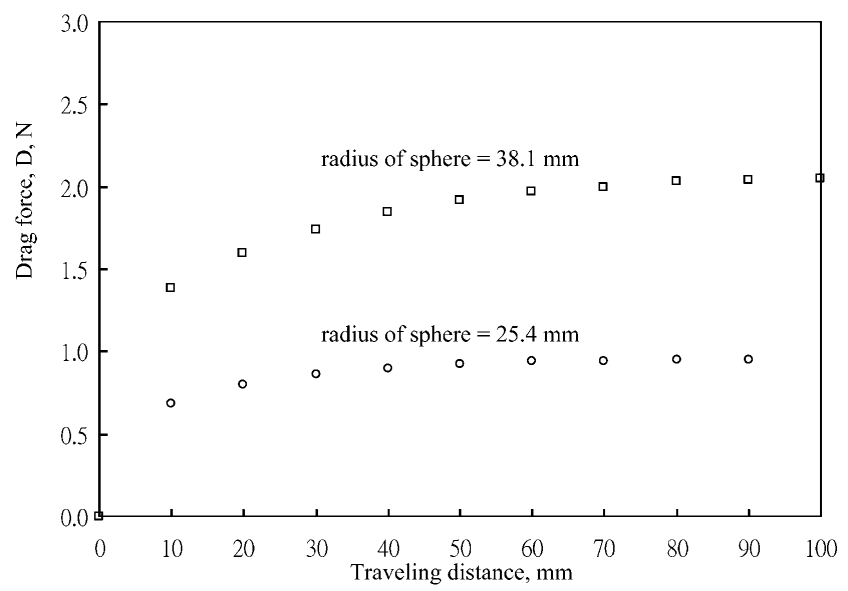

Fig. 10. Data of drag force vs. traveling distance of a sphere being dragged in clay grout $($ drag velocity $=0.5 \mathrm{~mm} / \mathrm{sec}$, water concentration of clay grout $=104 \%$ by weight $)$.

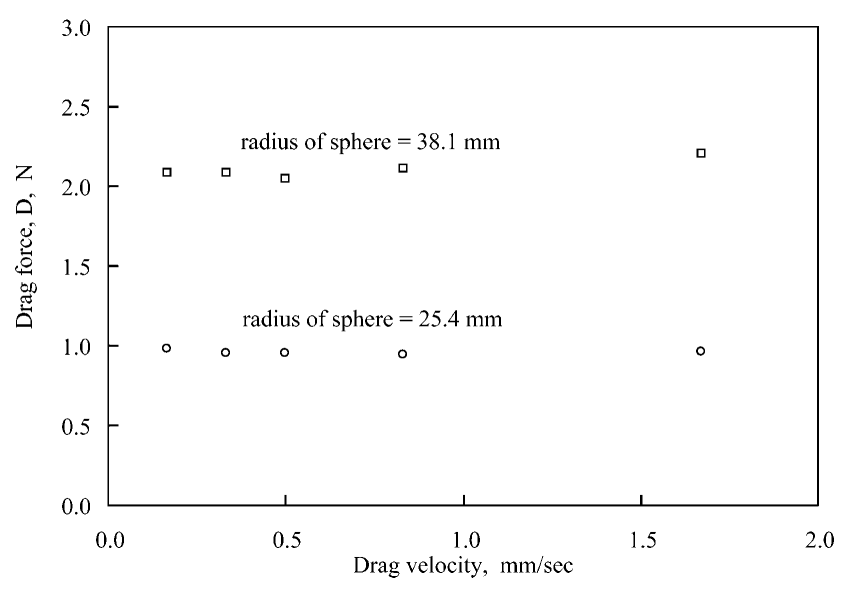

Fig. 11. Drag force vs. drag velocity of a sphere being dragged in clay grout (water concentration of clay grout $=104 \%$ by weight).

coarse and fine aggregates.

Figures $(10,12,14)$ show typical data of drag force versus traveling distance for the sphere as it is dragged upwards. In each experiment the drag force first increased monotonically at the beginning, and finally plateaued upon reaching the stable drag force corresponding to the drag velocity. Figures $(11,13,15)$ show some typical data for drag forces versus drag velocities where each data point stands for a single experiment

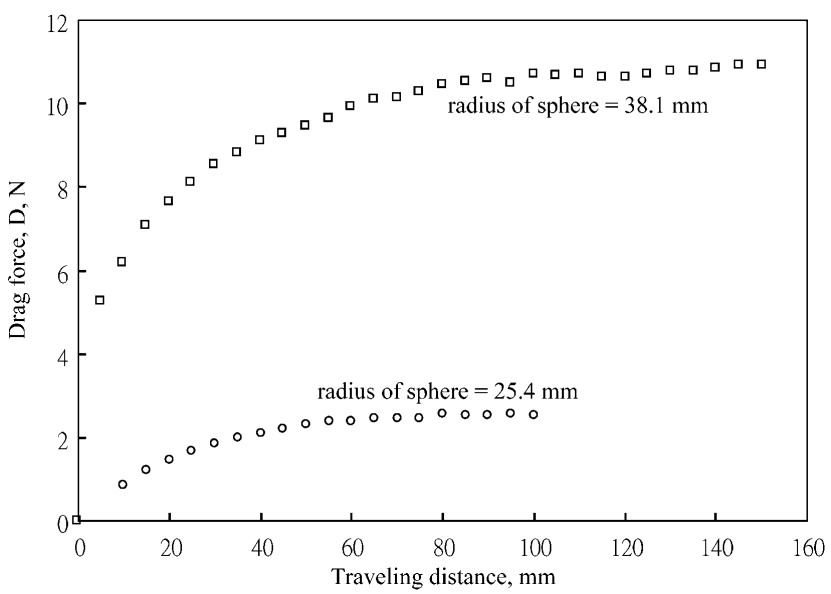

Fig. 12. Data of drag force vs. traveling distance of a sphere being dragged in mortar (drag velocity $=0.833 \mathrm{~mm} / \mathrm{sec}$, F.M. of fine aggregate $=3.1$, weight ratio of water, cement, and fine aggregate $=14.4 \%, 23.2 \%, 62.2 \%)$.

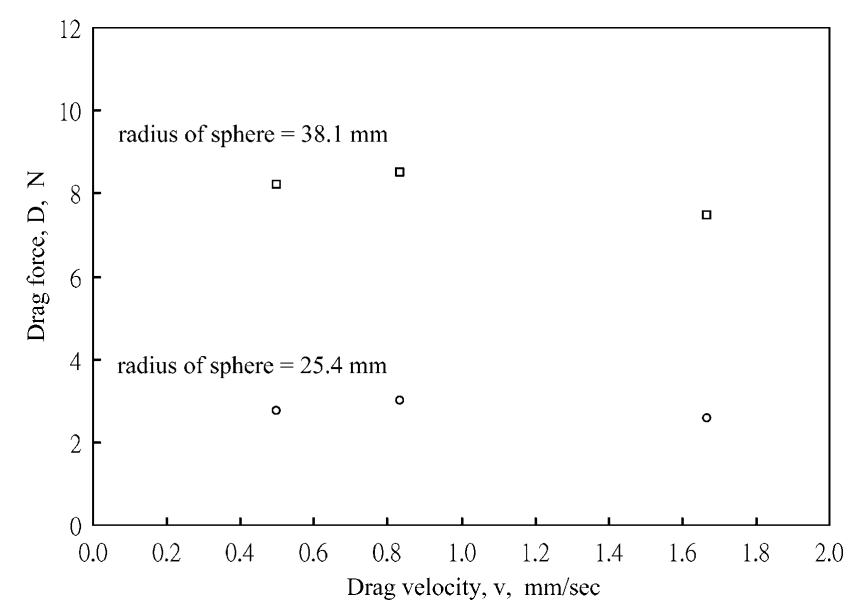

Fig. 13. Drag force vs. drag velocity of a sphere being dragged in fresh mortar (F.M. of fine aggregate $=3.1$, weight ratio of water, cement, and fine aggregate $=14.4 \%, 23.2 \%, 62.2 \%$ ).

with a specific drag velocity. The corresponding Reynolds number calculated from Eq. (10) was always in the order of $10^{-5}$ for all the experiments.

\section{ANALYSIS AND RESULTS}

The results in this work showed that the drag 
forces on the sphere during dragging in the clay grout and fresh mortar, were almost independent of drag velocity (Figs. 11, 13), indicating that clay grout and fresh mortar are not typical Bingham fluids, but are Bingham fluids with very small, if not zero, viscosity $\mu$. The yield shear stresses $\tau_{o}$, of the tested clay grout and the fresh mortar calculated from experimental the results of at least five experiments are $151 \pm 10 \mathrm{~Pa}$ and $506.9 \pm 30 \mathrm{~Pa}$, respectively.

Normal fresh concrete is much more complex in comparison with clay grout and mortar. With slow drag velocity, the drag force is linearly related to drag velocity. In this stage, fresh concrete does behave like a typical Bingham fluid. In the second stage, the drag velocity stars to decline swiftly as drag velocity exceeds a particular threshold. This second stage is a transition stage, during which the decline is limited. With continued increase in drag velocity, the drag force eventually reaches its lower bound. In the last stage, the drag force raises slowly as drag velocity increases. In this last stage, fresh concrete behaves like another Bingham fluid with relatively lower viscosity in comparison with that in the first stage.

Let $\mu, \tau_{o 1}, \mu_{2}$ and $\tau_{o 2}$ denote the viscosities and yield shear stresses of the fresh concrete during the first and last stages. In the transition stage, the fresh concrete behaves like a mix of both Bingham models. To simulate the experimental results (Fig. 15), the drag force is assumed to be,

$$
\begin{aligned}
D & =\left(6 \pi a \mu_{1} v+\pi^{2} a^{2} \tau_{o 1}\right) e^{-c v} \\
& +\left(6 \pi a \mu_{2} v+\pi^{2} a^{2} \tau_{02}\right)\left(1-e^{-c v}\right)
\end{aligned}
$$

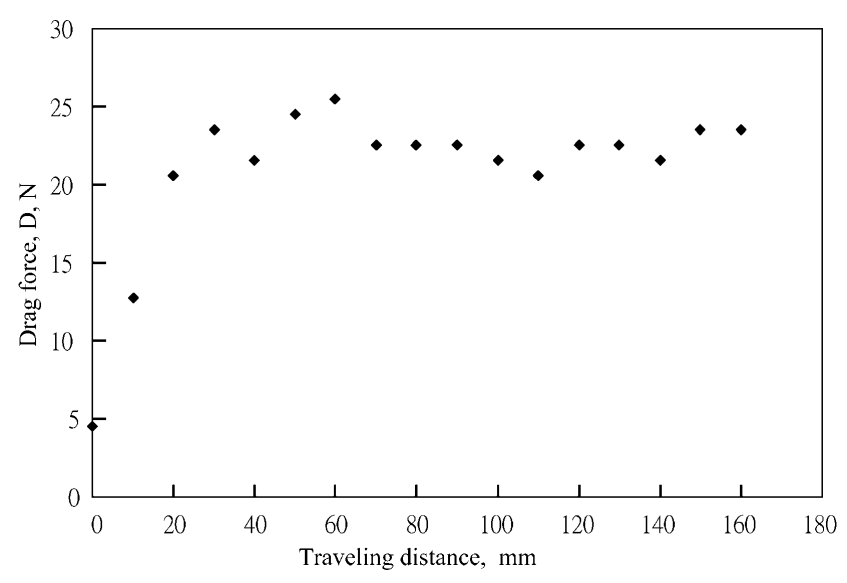

Fig. 14. Drag force vs. traveling distance of a sphere being dragged in fresh concrete (drag velocity $=0.833 \mathrm{~mm} / \mathrm{sec}$, F.M. of fine aggregate $=3.1$, dimension of coarse aggregate $=9.5 \mathrm{~mm}$, radius of sphere $=25.4 \mathrm{~mm}$, weight ratio of water, cement, fine aggregate, and coarse aggregate in the fresh concrete $\mathbf{= 1 . 4 \%}$, $12.2 \%, 40.2 \%, 40.2 \%)$.
Eq. (11) is designed to meet the conditions that when $v$ is small, $e^{-c v}$ is close to 1 , and,

$$
D \rightarrow 6 \pi a \mu_{1} v+\pi^{2} a^{2} \tau_{o 1}
$$

, and when $v$ is big, $e^{-c v}$ is close to 0 , and,

$$
D=6 \pi a \mu_{2} v+\pi^{2} a^{2} \tau_{02}
$$

Equation (11) indicates that the behavior of the specimen transfers from one Bingham model to another Bingham model as flow velocity increases, and the $c$ is the parameter controlling the transferring rate which is a material constant. Rearrange Eq. (11) to take the form as Eq. (3),

$$
\begin{aligned}
D & =6 \pi a\left[\mu_{1} e^{-c v}+\mu_{2}\left(1-e^{-c v}\right)\right] v \\
& +\pi^{2} a^{2}\left[\tau_{o 1} e^{-c v}+\tau_{02}\left(1-e^{-c v}\right)\right]
\end{aligned}
$$

and define the $\mu$ and $\tau_{o}$ in the transition stage as,

$$
\begin{aligned}
& \mu=\mu_{1} e^{-c v}+\mu_{2}\left(1-e^{-c v}\right) \\
& \tau_{0}=\tau_{o 1} e^{-c v}+\tau_{o 2}\left(1-e^{-c v}\right)
\end{aligned}
$$

When $v$ is small, $\mu$ is close to $\mu_{1}$ and $\tau_{o}$ is close to $\tau_{o 1}$. When $v$ is big enough, $\mu$ is close to $\mu_{2}$ and $\tau_{o}$ is close to $\tau_{o 2}$.

Let $D_{i}^{\prime}$ and $D_{i}$ represent the experimental and calculated $D$ from Eq. (11) or Eq. (14) when $v=v_{i}$. The error between $D_{i}^{\prime}$ and $D_{i}$ is,

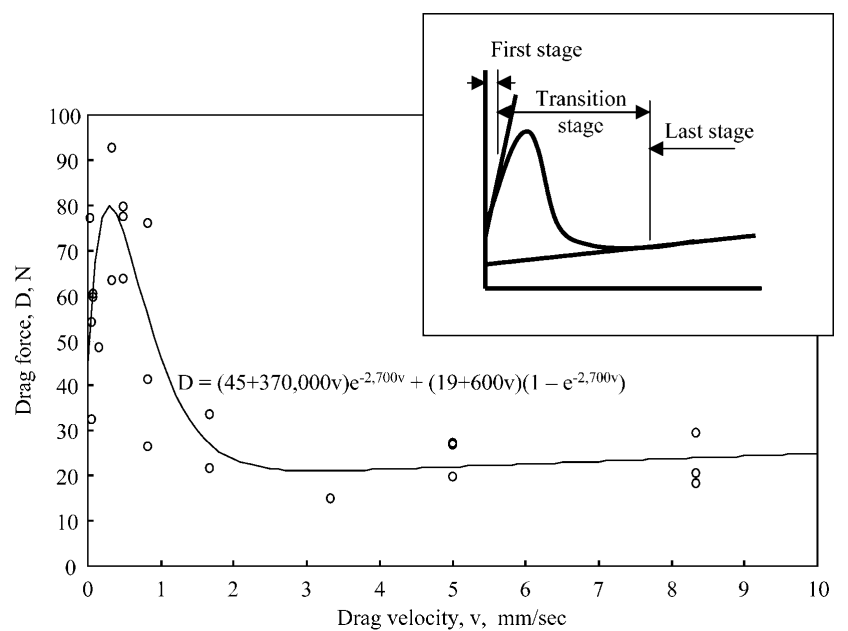

Fig. 15. Drag force vs. drag velocity of a sphere being dragged in fresh concrete and curve fitting (F.M. of fine aggregate $=3.1$, dimension of coarse aggregate $=9.5 \mathrm{~mm}$, radius of sphere $=25.4 \mathrm{~mm}$, weight ratio of water, cement, fine aggregate, and coarse aggregate in the fresh concrete $=7.4 \%, 12.2 \%, 40.2 \%, 40.2 \%$ ). 


$$
e_{i}=D_{i}^{\prime}-D_{i}
$$

Given $n$ data points, the summation of the error squares is,

$$
S=\sum_{i=1}^{n} e_{i}^{2}
$$

Based on the least squares curve fitting theory, the solutions of $c, \mu_{1}, \tau_{o 1}, \mu_{2}$ and $\tau_{o 2}$ in Eq. (14) are those that minimize $S$. The derivatives of $S$ with respect to $c$, $\mu_{1}, \tau_{o 1}, \mu_{2}$ and $\tau_{o 2}$ are taken and set to be zeros for calculating $c, \mu_{1}, \tau_{o 1}, \mu_{2}$ and $\tau_{o 2}$.

$$
\left[\begin{array}{l}
0 \\
0 \\
0 \\
0 \\
0
\end{array}\right]=\left[\begin{array}{c}
\partial S / \partial c \\
\partial S / \partial \mu_{1} \\
\partial S / \partial \tau_{01} \\
\partial S / \partial \mu_{2} \\
\partial S / \partial \tau_{02}
\end{array}\right]=-2\left[\begin{array}{c}
\sum_{i=1}^{n}\left(D_{i}^{\prime}-D_{i}\right) \partial D_{i} / \partial c \\
\sum_{i=1}^{n}\left(D_{i}^{\prime}-D_{i}\right) \partial D_{i} / \partial \mu_{1} \\
\sum_{i=1}^{n}\left(D_{i}^{\prime}-D_{i}\right) \partial D_{i} / \partial \tau_{01} \\
\sum_{i=1}^{n}\left(D_{i}^{\prime}-D_{i}\right) \partial D_{i} / \partial \mu_{2} \\
\sum_{i=1}^{n}\left(D_{i}^{\prime}-D_{i}\right) \partial D_{i} / \partial \tau_{02}
\end{array}\right]
$$

or

$$
\left[\begin{array}{l}
0 \\
0 \\
0 \\
0 \\
0
\end{array}\right]=\left[\begin{array}{c}
\sum_{i=1}^{n}\left(D_{i}^{\prime}-D_{i}\right) \partial D_{i} / \partial c \\
\sum_{i=1}^{n}\left(D_{i}^{\prime}-D_{i}\right) \partial D_{i} / \partial \mu_{1} \\
\sum_{i=1}^{n}\left(D_{i}^{\prime}-D_{i}\right) \partial D_{i} / \partial \tau_{01} \\
\sum_{i=1}^{n}\left(D_{i}^{\prime}-D_{i}\right) \partial D_{i} / \partial \mu_{2} \\
\sum_{i=1}^{n}\left(D_{i}^{\prime}-D_{i}\right) \partial D_{i} / \partial \tau_{02}
\end{array}\right]
$$

where

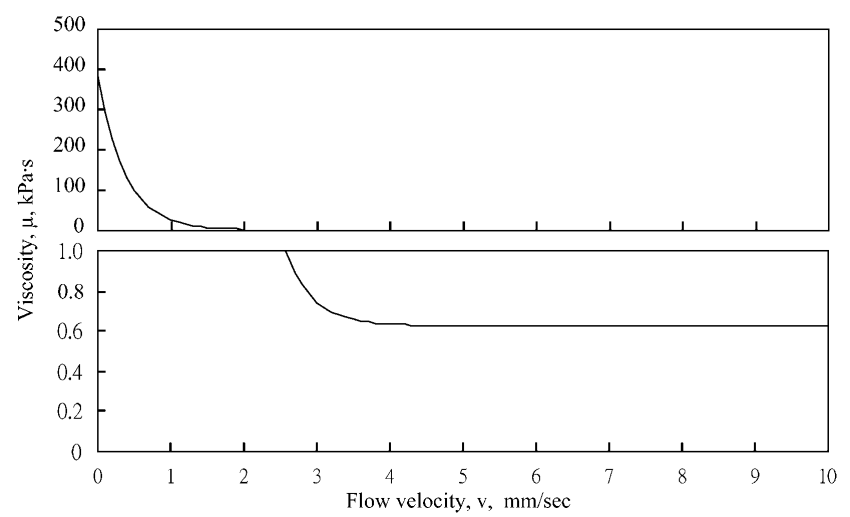

Fig. 16. Viscosity vs. flow velocity of fresh concrete (F.M. of fine aggregate $=3.1$, dimension of coarse aggregate $=9.5 \mathrm{~mm}$, radius of sphere $=\mathbf{2 5 . 4} \mathbf{m m}$, weight ratio of water, cement, fine aggregate, and coarse aggregate in the fresh concrete $=7.4 \%, 12.2 \%, 40$. $2 \%, \mathbf{4 0 . 2 \% )}$.

$$
\begin{aligned}
& \partial D_{i} / \partial c=\left[6 \pi a\left(\mu_{2}-\mu_{1}\right) v_{i}+\pi^{2} a^{2}\left(\tau_{o 2}-\tau_{o 1}\right)\right] v_{i} e^{-c v_{i}} \\
& \partial D_{i} / \partial \mu_{1}=6 \pi a v_{i} e^{-c v_{i}} \\
& \partial D_{i} / \partial \tau_{01}=\pi^{2} a^{2} e^{-c v_{i}} \\
& \partial D_{i} / \partial \mu_{2}=6 \pi a v_{i}\left(1-e^{-c v_{i}}\right) \\
& \partial D_{i} / \partial \tau_{02}=\pi^{2} a^{2}\left(1-e^{-c v_{i}}\right)
\end{aligned}
$$

Combine Eqs. (20-25) and simplify to obtain,

$$
\left[\begin{array}{l}
0 \\
0 \\
0 \\
0 \\
0
\end{array}\right]=\left[\begin{array}{c}
\sum_{i=1}^{n}\left(D_{i}^{\prime}-D_{i}\right)\left[6 \pi a\left(\mu_{2}-\mu_{1}\right) v_{i}+\pi^{2} a^{2}\left(\tau_{o 2}-\tau_{o 1}\right)\right] v_{i} e^{-c v_{i}} \\
\sum_{i=1}^{n}\left(D_{i}^{\prime}-D_{i}\right) v_{i} e^{-c v_{i}} \\
\sum_{i=1}^{n}\left(D_{i}^{\prime}-D_{i}\right) e^{-c v_{i}} \\
\sum_{i=1}^{n}\left(D_{i}^{\prime}-D_{i}\right) v_{i}\left(1-e^{-c v_{i}}\right) \\
\sum_{i=1}^{n}\left(D_{i}^{\prime}-D_{i}\right)\left(1-e^{-c v_{i}}\right)
\end{array}\right]
$$

Equation (26) can be solved using the NewtonRaphson iteration method to obtain the $c, \mu_{1}, \tau_{o 1}, \mu_{2}$ and $\tau_{o 2}$. From the experimental data of drag force versus drag velocity such as Fig. 15 , the c, $c, \mu_{1}, \tau_{o 1}, \mu_{2}$ and $\tau_{o 2}$ are calculated to be $2,700 \tilde{\mathrm{Nb}} 100 \mathrm{~s} / \mathrm{m}, 386,400 \pm 5,000$ $\mathrm{Pa} \bullet \mathrm{s}, 1,767 \pm 200 \mathrm{~Pa}, 627 \pm 100 \mathrm{~Pa} \bullet \mathrm{s}$, and $746 \pm 100 \mathrm{~Pa}$. The $\mu$ and $\tau_{o}$ calculated by Eqs. $(15,16)$ are shown in Figs. $(16,17)$. The calculation results indicates that, the viscosity $\mu$ rapidly decreases from $386,400 \mathrm{~Pa} \bullet \mathrm{s}\left(=\mu_{1}\right)$

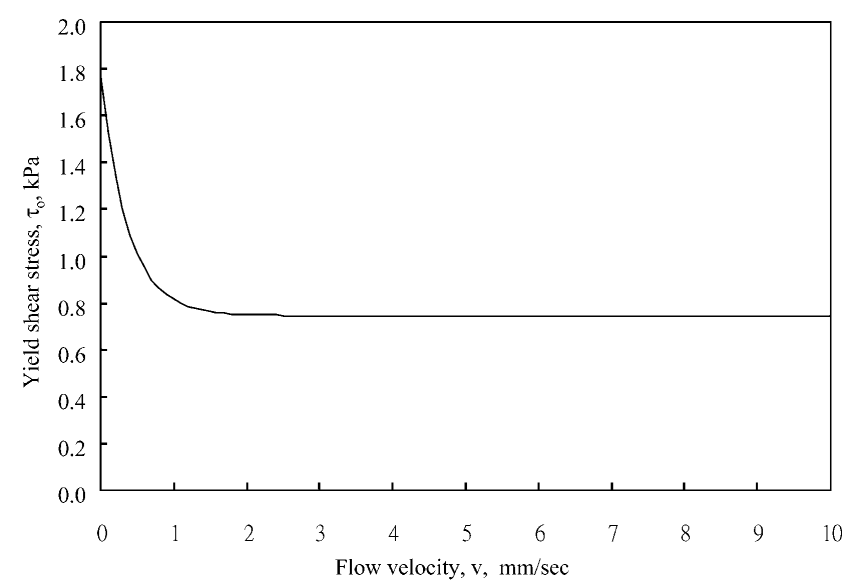

Fig. 17. Yield shear stress vs. flow velocity of fresh concrete (F.M. of fine aggregate $=3.1$, dimension of coarse aggregate $=9.5 \mathrm{~mm}$, radius of sphere $=\mathbf{2 5 . 4} \mathbf{m m}$, weight ratio of water, cement, fine aggregate, and coarse aggregate in the fresh concrete $=\mathbf{7 . 4 \%}$, $12.2 \%, 40.2 \%, 40.2 \%$ ). 


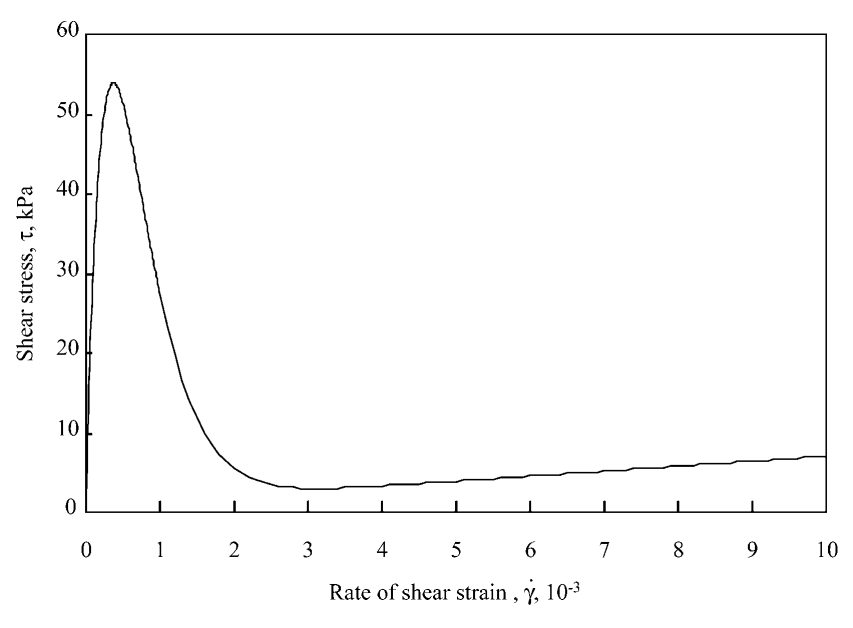

Fig. 18. Shear stress vs. rate of shear strain of fresh concrete (F.M. of fine aggregate $=3.1$, dimension of coarse aggregate $=9.5 \mathrm{~mm}$, radius of sphere $\mathbf{=} \mathbf{2 5 . 4} \mathrm{mm}$, weight ratio of water, cement, fine aggregate, and coarse aggregate in the fresh concrete $=\mathbf{7 . 4 \%}$, $12.2 \%, 40.2 \%, 40.2 \%$ ).

to $627 \mathrm{~Pa} \cdot \mathrm{s}\left(=\mu_{2}\right)$, and thereafter remains constant (Fig. $16)$, and the yield shear stress $\tau_{o}$ decreases swiftly from $1,767 \mathrm{~Pa}\left(=\tau_{o 1}\right)$ to $746 \mathrm{~Pa}\left(=\tau_{o 2}\right)$, and consequently remains constant (Fig. 17). The shear stress calculated from Eq. (1) versus rate of shear strain is shown in Fig. (8). The calculation results indicate clearly that, the tested fresh concrete behaves like a tough Bingham fluid when $v$ is lower than $0.3 \mathrm{~mm} / \mathrm{s}$, softens when $v$ ranges between 0.3 and $3 \mathrm{~mm} / \mathrm{s}$, starts to behaves like another Bingham fluid with much lower viscosity in comparison with that in the first stage when $v$ is over 3 $\mathrm{mm} / \mathrm{s}$.

\section{DISCUSSIONS}

Experimental results indicate that flows of clay grout and fresh mortar are almost perfectly plastic. Yield shear stress dominates the rheological behaviors of such flows. Meanwhile, viscosity exerts only a very minor influence.

The results also indicate that the viscosity and yield shear stress of fresh concrete are not constants but rather depending on flow velocity contradicting the basic assumption of Eq. (3) that they are constant [9]. Consequently, the viscosity É $`$ Éz and yield shear stress $\tau_{o}$, obtained then are approximate rather than exact. If viscosity and yield shear stress are assumed to depend on flow velocity at very beginning, deriving Eq. (3) becomes extremely difficult because the exact local flow velocity of the fluid sliding along the sphere surface is variable. Since the fitting of the theoretical prediction of drag force versus drag velocity to the experimental results is excellent, the assumption of constant viscosity and yield shear stress is acceptable.

Several researchers have verified the shearthinning, reduction of viscosity, of fresh concrete subjected to vibration, using apparatuses like viscometers $[1,2,5,7,9-11]$. The experimental results presented here imply another sort of shear-thinning phenomenon involving fresh concrete, induced by increasing the flow velocity during the transition stage. The tested normal fresh concrete exhibited a critical flow velocity of $0.3 \mathrm{~mm} / \mathrm{s}$. The temporary internal aggregate structure of the concrete resists the shear stress when the flow velocity is smaller than the critical velocity. Concrete that acts like a tough Bingham fluid is not really flowing but rather slowly deforming. When the flow velocity exceeds the critical velocity, the shear resistance of the concrete declines significantly, implying destruction of the temporary internal structure before the start of a real flow. The destruction of the internal structure ends at $v=3 \mathrm{~mm} / \mathrm{s}$, and the concrete starts to flow like a real fluid, a much softer Bingham fluid in comparison with that when the flow velocity is low.

The shear stress induced by pushing pressure must exceed than the shear stress of the concrete during the last stage to maintain the flow. The shear stress calculated from Eq. (1) based on above results is shown in Fig. 18, which indicates that, the shear stress required to start a flow must exceed the peak shear stress between the first and the transition stages, which is higher than the value required for maintaining the flow. The increasing shear stress at low rate of shear strain stands for the resisting of the temporary internal aggregate structure to the external shear stress.

\section{CONCLUSIONS}

The proposed method can effectively characterize the rhelogical behaviors of normal fresh concrete, maltose, clay grout and fresh mortar. The main contribution of this study is establishing an algorithm and process for characterizing fluid viscosity and shear yield stress, especially for normal fresh concrete. This study verified that clay grout and cement mortar are Bingham fluids with very small viscosities. This study also clarified the transition phenomenon of fresh concrete from a Bingham model to another Bingham model, and verified that the viscosity and shear yield stress of normal fresh concrete depend on flow velocity especially when the flow is slow. Still, more experiments are required in the future for more detailed study of concrete flow, to address the effects of admixtures, ingredient mix ratios and environmental conditions. 


\section{NOTATIONS}

A

frontal area of a sphere normal to flowing direc-

tion

radius of a sphere

$C_{D} \quad$ drag coefficient

$c$ parameter in decaying function $e^{-c v}$

$D$ pushing force or drag force

$D_{i}^{\prime} \quad$ experimental $D$ when drag velocity is $v_{i}$

$D_{i} \quad$ calculated $D$ when drag velocity is $v_{i}$

$e_{i} \quad D_{i}^{\prime}-D_{i}$

$G \quad$ gravity

$n \quad$ number of data

$R \quad$ Reynolds number

$S \quad \sum_{i=1}^{n} e_{i}^{2}$

$v$

W

$\alpha$

$\dot{\gamma}$

$\mu$

$\mu_{1}$

$\mu_{2}$

$\rho$

$\rho_{s}$

$\tau$

$\tau_{o}$

$\tau_{o 1} \quad$ yield shear stress of concrete when flowing rate is low

$\tau_{o 2}$ yield shear stress of concrete when flowing rate is high

\section{REFERENCES}

1. Daily, J.M. and Harleman, D.R.F., Fluid Dynamics, Addison-Wesley Publishing Co. Inc. (1966).

2. Domone, P.L.J., Xu, Yongmo, and Banfill, P.F.G., "Developments of The Two-Point Workability Test for
High-Performance Concrete," Mag. Concrete Res., Vol. 51, pp. 171-179 (1999).

3. Hu, Chong and Larrard, F., "The Rheology of Fresh High-Performance Concrete," Cement Concrete Res., Vol. 26, pp. 283-294 (1996).

4. Japanese Society of Civil Engineers, Standard Concrete Specification (Work Edition), JSCE, Tokyo, Japan, pp. 259-262 (1992).

5. Kahuta, S. and Kojima, T., "Rheology of Fresh Concrete under Vibration," Rheology of Fresh Cement and Concrete, Proceedings of the International Conference, ed. by Banfill, P.F.G., University of Liverpool, UK, Mar. 16-29, Chapman and Hall, London, pp. 339-342 (1990).

6. Kred, R.D. and Walker, R.D., Highway Material, McGraw Hill, New York, pp. 331-380 (1971).

7. Larrard, F., Hu, C., Sedran, T., Szitkar, J.C., Joly, M., Claux, F., and Derkx, F., "A New Rheometer for Soft-toFluid Fresh Concrete," ACI Mat. J., Vol. 94, No. 3, pp. 234-243 (1997).

8. Murata, J. and Suzuki, K., "New Method of Test the Flowability of Grout," Mag. Concrete Res., Vol. 49, pp. 269-276 (1997).

9. Petrou, Michael F., Wan, Baolin, Gardala-Maria, Francis, Kolli, Venkata Giri, and Harries, Kent A., "Influence of Mortar Rheology on Aggregate Settlement," ACI Mat. J., No. 97-M56, pp. 479-485 (2000).

10. Tattersall, G.H., "Effect of Vibration on the Rheological Properties of Fresh Cement Pastes and Concretes," Rheology of Fresh Cement and Concrete, Proceedings of the International Conference, P.F.G. Banfill, ed., University of Liverpool, UK, Mar. 16-29, Chapman and Hall, London, pp. 323-338 (1990).

11. Tattersall, G.H. and Baker, P.H., "The Effect of Vibration on the Rheological Properties of Fresh Concrete," Mag. Concrete Res., Vol. 40, No. 13, pp. $79-89$ (1998).

12. Tattersall, G.H. and Banfill, P.F.G., The Rheology of Fresh Concrete, Pitman Advanced Publishing Program, London (1983).

13. Zhang, Xiong and Han, Jihong, "The Effect of Ultrafine Admixture on the Rheological Property of Cement Paste," Cement Concrete Res., Vol. 30, pp. 827-830 (2000). 